\title{
Infektionen in der Schwangerschaft
}

Jan Pauluschke-Fröhlich

Nicht erst seit der Corona-Pandemie sind Infektionen in der Schwangerschaft ein relevantes Thema. Die Mutterschafts-Richtlinien erfassen eine Reihe von Untersuchungen, es gibt jedoch auch Infektionen, die nicht durch das Routine-Screening abgedeckt sind, aber die Gesundheit von Mutter und Kind gefährden. Dr. med. Jan Pauluschke-Fröhlich vermittelte beim Forum Hebammenarbeit im März 2020 in Leipzig, was Hebammen und Mediziner im Blick haben müssen und wie sie werdende Mütter bzw. Eltern kompetent beraten.

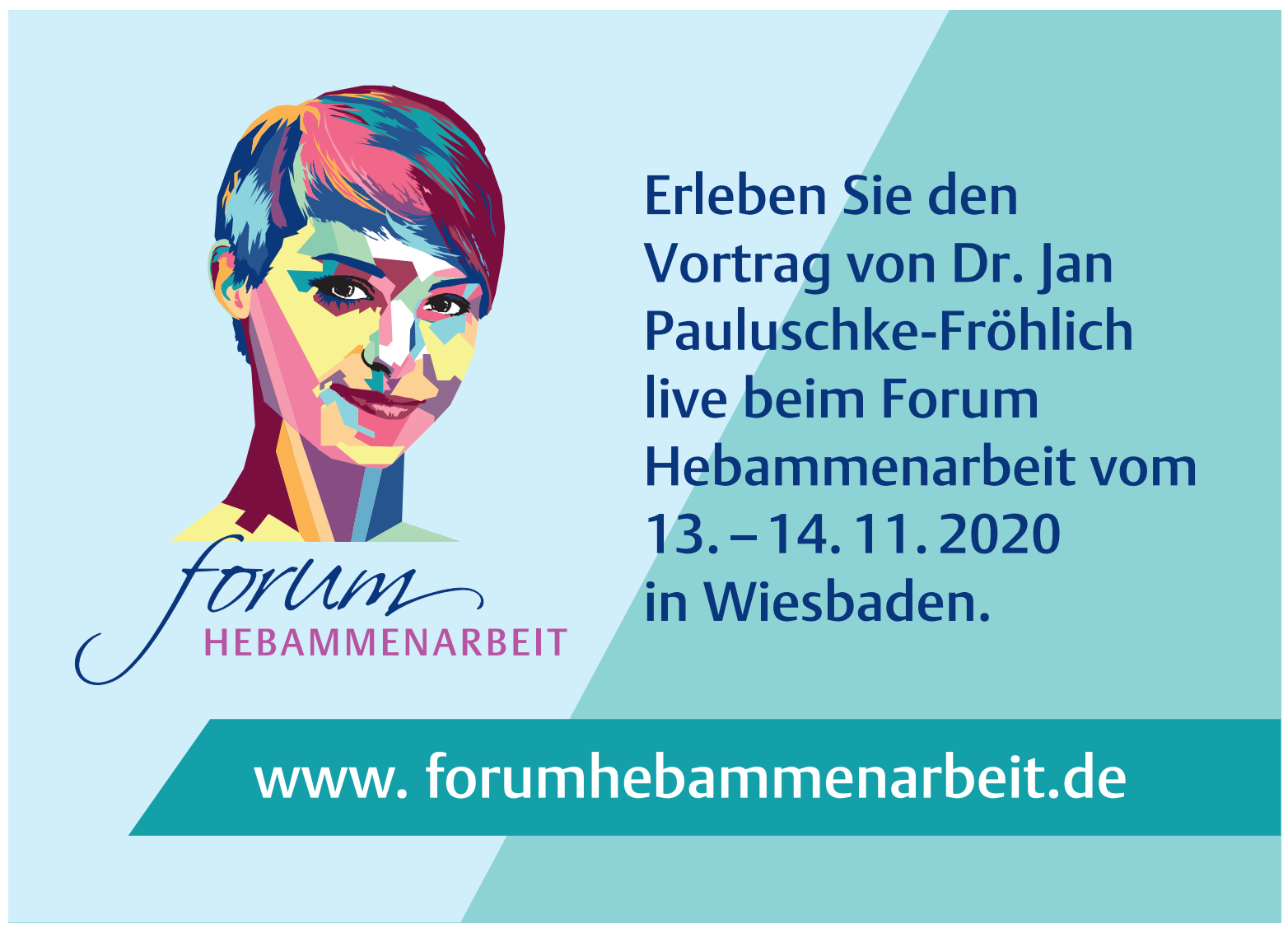

Relevante Infektionen und Tests in der Schwangerschaft

Die Mutterschafts-Richtlinien sehen im Rahmen der Vorsorgeuntersuchungen Screenings u. a. auf Hepatitis B, Röteln, Chlamydien und Lues vor. Eine Reihe ebenso relevanter Infektionen ist jedoch nicht abgedeckt ( Tab. 1).

\section{Chlamydien}

Chlamydien sind die am häufigsten sexuell übertragenen Bakterien in Europa und den USA. Bei der Frau führen sie in ihrer häufigsten Form zu einer Adnexitis, einer Entzündung von Eileiter, Eierstock und umgebendem Gewebe, was die Fruchtbarkeit negativ beeinflussen kann. In Deutschland sind rund 100000 Frauen von einer Chlamydien-assoziierten Sub-Infertilität betroffen. 
- Tab. 1 Infektionen in der Schwangerschaft-Tests in der Schwangerenvorsorge

\begin{tabular}{|l|l|l|l|}
\hline & Viren & $\begin{array}{l}\text { Aszendie- } \\
\text { rende } \\
\text { Infektionen }\end{array}$ & $\begin{array}{l}\text { Bakterien / } \\
\text { Protozoen }\end{array}$ \\
\hline $\begin{array}{l}\text { Test verbind- } \\
\text { lich } \\
\text { vorgesehen }\end{array}$ & $\begin{array}{l}\text { Hepatitis B } \\
\text { Röteln }\end{array}$ & Chlamydien & Lues \\
\hline $\begin{array}{l}\text { Test muss } \\
\text { angeboten } \\
\text { werden }\end{array}$ & HIV & & \\
\hline kein Test & $\begin{array}{l}\text { CMV } \\
\text { Parvovirus } \\
\text { B19 } \\
\text { Varizellen } \\
\text { Hepatitis C }\end{array}$ & $\begin{array}{l}\text { B-Streptokok- } \\
\text { ken }\end{array}$ & $\begin{array}{l}\text { Toxoplasmose } \\
\text { Listerien }\end{array}$ \\
\hline Quelle: nach Mutterschafts-Richtlinien [2] & \\
\hline
\end{tabular}

Schwangerschaftsrisiken bei Chlamydien:

- Cervicitis mit hohem Risiko für vorzeitigen Blasensprung

- vorzeitige Wehen

- Frühgeburtlichkeit

- neonatale Infektion während der Geburt (50-70\%)

- Konjunktivitis

- Pneumonie

Therapie: Bei positivem Nachweis im Rahmen der Schwangerenvorsorge sind Chlamydien konsequent zu behandeln (Erythromycin, Azithromycin, Ofloxacin, Doxycyclin). Wichtig ist, dass auch der Partner der Frau sich einer Behandlung dieser sexuell übertragbaren Krankheit unterziehen muss.

\section{Röteln}

Das Rötelnvirus (Rubella virus) löst die hochansteckende und durch Tröpfcheninfektion übertragbare Rötelnkrankheit aus. Gefährdung und Ausmaß sind abhängig vom Gestationsalter: 4.-12. SSW 85-90\% und ab 2. Trimester $25-30 \%$.

\section{Gregg-Syndrom und allgemeine Folgen:}

- Augenfehlbildungen

- Herzfehlbildungen

- Mikrocephalie und Innenohrschäden

- Kind blind, taub, mental retardiert

- 10-20\% Letalität im 1. Lebensjahr

\section{Historie:}

- USA 1964/ 65 Rötelnepidemie

- 11.000 Röteln-induzierte Aborte

- 20.000 konnatales Röteln-Syndrom

Realität: BRD 2001-2010 10 konnatal infizierte Kinder
Prävention: Die Impfung bietet Schutz vor Röteln. Bei Schwangeren, die zwei erfolgte Impfungen nachweisen können, sind keine weiteren Tests notwendig.

Test: Überprüfung über Hämagglutinationshemmtest (HAH)

- sicherer Schutz ab 1:16

- Impfung in der Schwangerschaft kontraindiziert

- Bisher sind jedoch keine teratogenen Effekte beschrieben

\section{Hepatitis B}

Weltweit sind ca. 200-350 Mio. Menschen von Hepatitis B betroffen. Ähnlich wie HIV wird das Virus HBV durch Körperflüssigkeiten übertragen, v. a. beim ungeschützten Geschlechtsverkehr oder gemeinsamer Benutzung von Injektionsbesteck. Bei 10-15\% der akut Erkrankten verläuft die Krankheit chronisch.

Die akute Hepatitis in der Schwangerschaft verläuft in der Regel mild. Es besteht kein Potenzial für eine Fruchtschädigung. Die Therapie ist in der Regel rein supportiv. Die Rate für die Übertragung von der Mutter aufs Kind beträgt bei Infektion in der Frühschwangerschaft $10 \%$. Um die Geburt liegt die Transmissionsrate allerdings bei bis zu $60 \%$.

In 10-15\% der Fälle verläuft die Hepatitis-B-Infektion chronisch. Bei neonataler Infektion sind $50 \%$ chronische Verläufe zu beobachten. Es besteht in diesem Fall zudem eine höhere Rate an Progressen zur Leberzirrhose und zum Hepatozellulären Karzinom.

Verlauf der Hepatitis B in der Schwangerschaft: Akute Schübe (Erhöhung der GPT auf das 2-3-fache) sind mit ca. $2 \%$ selten. CAVE post partum kommt es bis zu $25 \%$ zu Krankheitsschüben.

Prävention: Eine Impfung schützt vor Hepatitis B. Stillen ist nach der Impfung erlaubt. Muttermilchspende ist allerdings nicht möglich.

Test:

- Bei Nachweis des HBs-Antigens ist eine Infektiosität anzunehmen.

- Bei Nachweis von Anti-HBs-Antikörpern bei der Mutter nach erfolgter Infektion oder Impfung besteht ein sicherer Schutz auch für das Kind.

- Ist die Mutter HBs-Antigen positiv oder hat sie einen unbekannten Status, ist eine Aktiv-Passiv-Impfung des Neugeborenen indiziert.

- Bei hoher Viruslast ist eine Therapie z.B. mit Tenofovir vor der 32. SSW erforderlich (HBV-DNA > 200000 IE/ $\mathrm{ml}$ oder $>1000000$ Kopien/ml bei unauffälligem Labor).

Prophylaxe: Das Kind wird aktiv und passiv innerhalb von $12 \mathrm{~h}$ pp geimpft. Die Transmissionsrate nativ bei 
chronischer Hepatitis liegt bei $90 \%$. Die Transmissionsrate bei Immunisierung des Neugeborenen innerhalb von $12 \mathrm{~h}$ pp liegt bei $1,1 \%$.

Geburtshilfliches Management: Bei Müttern mit Hepatitis B liegt für das Vorgehen bei vorzeitigem Blasensprung (PROM = Prematuere Rupture Of Membranes) derzeit keine eindeutige Datenlage vor. Daher wird kein abweichendes Prozedere bei der Geburt empfohlen. Es besteht kein Vorteil für die Sectio gegenüber vaginaler Geburt. Bei hoher Viruslast sollte auf vaginal operative Entbindung verzichtet werden.

\section{Hepatitis C}

Die Transmissionsrate für Hepatitis C liegt etwa bei 5-6\%, bei Co-Infektion mit HIV ist sie etwas erhöht mit $11 \%$. Bei PROM sollte die Geburt nicht prolongiert werden. Der Geburtsmodus selbst hat aber keinen Einfluss auf die Transmissionsrate. Müttern mit Hepatitis $C$ ist grundsätzlich das Stillen erlaubt. Bei einer aktiven Hepatitis C sollte aber darauf verzichtet werden.

\section{HIV}

Seit 1987 ist der HIV-Test Teil der Mutterschaftsvorsorge. Es besteht die Pflicht, über die Folgen einer HIV-Infektion für die Schwangerschaft aufzuklären und den Test anzubieten. Ca. ein Drittel der HIV-Erstdiagnosen bei Frauen werden im Rahmen der Schwangerschaftsvorsorge gestellt. Für zwei Drittel davon ist das positive Ergebnis unerwartet.

\section{Maternale Komplikationen bei HIV-Infektion:}

- Anämie

- Herpes genitalis

- genitale Candidosis

- HPV-Koinfektion (bis zu $30 \%$ !)

- Kondylome und zervikale Dysplasie/ Neoplasie

Ohne prophylaktische Maßnahmen kommt es in bis zu 40 \% der Fälle hämatogen über die Plazenta zur MutterKind-Transmission des HI-Virus. Weitere Transmissionswege sind Blut-Sekretkontakt während der Geburt und die Übertragung durch Muttermilch. Bei diagnostizierter HIV-Infektion und entsprechenden Interventionen kann die Transmissionsrate auf 1-2\% gesenkt werden.

\section{Prophylaxe der HIV-Transmission:}

- antiretrovirale Therapie in der Schwangerschaft

- Beginn abhängig von maternaler Symptomatik, Viruslast und CD4-Zahl

- peripartaler antiretroviraler Schutz (i. d. R. ZidovudinInfusion)

- primäre Sectio um $37+0$ SSW

- ggf. vaginale Geburt vertretbar, wenn Viruslast< 500 Kopien, besser wenn Viruslast unter Nachweisgrenze

- antivirale Therapie des Neugeborenen

- Stillverzicht
Merke

Infektionen mit Chlamydien, Röteln, Hepatitis und HIV in der Schwangerschaft können im Rahmen der Mutterschafts-Richtlinien eindeutig diagnostiziert werden. Es existieren wirksame präventive Maßnahmen und gute Therapiemöglichkeiten. Daher kommt es in Deutschland nur selten zu geschädigten Kindern.

\section{Fragen an den Experten}

In seinem Vortrag auf dem Forum Hebammenarbeit 2020 am 13. November 2020 in Wiesbaden wird Dr. Jan Pauluschke Fröhlich auch auf weitere Infektionen in der Schwangerschaft eingehen, die aktuell nicht in den Mutterschafts-Richtlinien vorgesehen sind, aber das maternale oder neonatale Outcome beeinflussen können. Behandelt werden u.a. die Infektion mit dem Cytomegalie-Virus (CMV) sowie die perinatale Herpes-simplex-Virus-Infektion (HSV), deren Therapieansätze und das geburtshilfliche Management. Ferner stellt er Effekte der B-Streptokokken-Prophylaxe vor. Zudem gibt der Experte ein Update zU SARS-CoV-2-Infektionen als Beitrag zur aktuellen Corona-Pandemie und deren Bedeutung für die Schwangerenberatung, Geburtshilfe und Wochenbettbetreuung (siehe hierzu auch Fachartikel in dieser Ausgabe, Seite 17). Wie immer besteht auch die Möglichkeit, nach dem Vortrag Fragen an den Referenten zu stellen und sich mit Kolleginnen und Kollegen sowie weiteren Expertinnen und Experten fachlich auszutauschen. Alle Infos zum Kongress unter www.forumhebammenarbeit.de

Autorinnen / Autoren

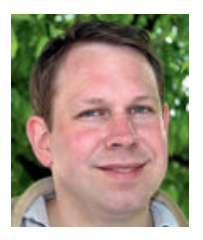

Dr. Jan Pauluschke-Fröhlich ist Perinatologe und oberärztlicher Leiter des Kreißsaals am Department für Frauengesundheit der Universitätsfrauenklinik Tübingen.

Anja Stamm ist freie Redakteurin in Frankfurt am Main und hat einige Aspekte aus dem Fachvortrag für diesen Artikel zusammengefasst.

\section{Literatur}

[1] Becker MA. Coronavirus: Was bisher über SARS-CoV-2 bekannt ist. Die Hebamme 2020; (3): 17-22

[2] Gemeinsamer Bundesausschuss. Mutterschafts-Richtlinien. Im Internet: https://www.g-ba.de/richtlinien/19/

\section{Bibliografie}

DOI https://doi.org/10.1055/a-1137-8562

Die Hebamme 2020; 33: 45-47

(C) Georg Thieme Verlag KG Stuttgart · New York ISSN 0932-8122 